\title{
Strategies to improve adherence to treatment in adolescents and young adults with cancer: a systematic review
}

This article was published in the following Dove Press journal:

Clinical Oncology in Adolescents and Young Adults

10 July 2015

Number of times this article has been viewed

\author{
Eden G Robertson ${ }^{1,2}$ \\ Claire E Wakefield ${ }^{1,2}$ \\ Kate H Marshall ${ }^{2}$ \\ Ursula M Sansom-Daly ${ }^{1-3}$ \\ 'Discipline of Paediatrics, School \\ of Women's and Children's Health, \\ UNSW Medicine, University of \\ New South Wales, Kensington, \\ NSW, Australia; ${ }^{2}$ Behavioural Sciences \\ Unit, Kids Cancer Centre, Sydney \\ Children's Hospital, ${ }^{3}$ Sydney Youth \\ Cancer Service, Prince of Wales/ \\ Sydney Children's Hospital, Randwick, \\ NSW, Australia
}

Purpose: Adolescents and young adults (AYAs) with cancer have higher rates of nonadherence to treatment relative to younger and older cancer patients. Efforts to improve adherence in this population are therefore increasing. This review aimed: 1) to synthesize recommendations and strategies used to improve treatment adherence in AYAs with cancer, and 2) to summarize the available evidence supporting the efficacy of adherence-promoting strategies for AYAs with cancer.

Methods: We conducted a systematic review with two stages: 1) a narrative stage, to analyze expert recommendations, and 2) an evaluative stage, to summarize quantitative evidence for interventions. Four electronic databases were searched for studies involving AYAs, aged 10-39 years, with cancer, published from 2005 to 2015. Preferred Reporting Items for Systematic Reviews and Meta-Analyses (PRISMA) guidelines were used to ensure quality of the review. The Delphi list was used to assess study quality.

Results: Nine articles were identified in the narrative stage of the review. For the evaluative stage, out of 113 screened abstracts, only one eligible intervention was identified. Common themes of adherence-promoting strategies were grouped into five domains: developmental, communication, educational, psychological well-being, and logistical/management strategies. Strategies to address developmental stage and to improve communication were the most highly recommended to improve adherence. Few strategies focused on the role of the patient in adherence. One intervention found that a behaviorally targeted computer game could significantly improve adherence to prescribed oral medication in AYAs with cancer.

Conclusion: Although numerous studies report challenges to treatment adherence in AYAs with cancer, little research has systematically evaluated the impact of implementing recommended strategies and interventions in this age group. The present review extends the current literature through its focus on strategies recommended to improve adherence, rather than focusing on barriers and risk factors for nonadherence. There is now a need for more rigorous research to systematically assess the effect of implementing strategies to improve AYAs' adherence to cancer treatment.

Keywords: neoplasms, emerging adulthood, interventions, communication, psychosocial

\section{Introduction}

Each year there are more than one million new cancer diagnoses worldwide among 15-39 year olds. ${ }^{1}$ Despite improved prognoses in this population, there remain a significant number of relapses and deaths. ${ }^{2,3}$ Nonadherence to treatment is one factor that may contribute to the lower rates of survival improvement among adolescents and young adults (AYAs), relative to other age groups. ${ }^{2,4,5}$ With the rise of efficacious selfadministered medications for a range of cancer diagnoses, ${ }^{6}$ it is increasingly important
Correspondence: Eden G Robertson Behavioural Sciences Unit proudly supported by the Kids with Cancer Foundation, Kids Cancer Centre, Sydney Children's Hospital, Level I, High St, Randwick, NSW 203I, Australia

Tel $+6 \mid 293823120$

Fax +6I 293821789

Email eden.robertson@unsw.edu.au
Clinical Oncology in Adolescents and Young Adults 20I5:5 35-49 35

Dovepress.

http://dx.doi.org/10.2147/COAYA.585988 (c) (i) (5) 2015 Robertson et al. This work is published by Dove Medical Press Limited, and licensed under Creative Commons Attribution - Non Commercial (unported, v3.0) permission from Dove Medical Press Limited, provided the work is properly attributed. Permissions beyond the scope of the License are administered by Dove Medical Press Limited. Information on permission from Dove Medical Press Limited, provided the work is properly attributed. how to request permission may be found at: http://www.dovepress.com/permissions.php 
to ensure that young patients are able to properly adhere to their treatment regimens. Despite this, suboptimal adherence to treatment, oral chemotherapy in particular, appears considerably more problematic in AYAs than in pediatric and adult/geriatric patients, ${ }^{5,7,8}$ with up to $60 \%$ of AYAs failing to adhere to the recommendations of their medical team. ${ }^{46,9}$

Adherence, also referred to as "compliance", is most commonly defined as "the extent to which a person's behavior (with regards to medication, diet, or lifestyle) corresponds with agreed recommendations from health care providers". ${ }^{10}$ In cancer, nonadherence may present as inconsistent medication use (including oral chemotherapy), failure to attend clinic appointments, and failure to engage in self-care behaviors. ${ }^{11}$ Researchers and clinicians define acceptable medication adherence as $80 \%$ or more. ${ }^{12}$

The impact of nonadherence to cancer treatment on survival in AYAs has not yet been fully explored. ${ }^{11,13}$ Medication nonadherence can increase relapse risk and reduce survival in pediatric cancer patients. ${ }^{14,15}$ It can also cause increased mortality among older populations, such as adult women with breast cancer, ${ }^{16}$ and can lead to hospitalization and death for cancer patients through overdosing. ${ }^{17}$ In the context of research, nonadherence can also invalidate results of trials, and prevent adequate evaluation of treatment efficacy. ${ }^{18}$

A variety of factors have been attributed to AYAs' poor adherence, including developmental factors, interpersonal/ support-related factors, and informational factors. Regarding developmental factors, AYAs with cancer are faced with unique challenges as they enter cancer treatment. AYAs may have underdeveloped coping skills and a more concrete thinking style than older adults. ${ }^{5}$ Factors such as reduced autonomy during treatment, an inability to conceive the long-term consequences of their illness, and emerging communication skills also have the potential to negatively affect adherence in this population. ${ }^{19,20}$

Across AYAs with other chronic illnesses, factors such as the desire for "normality" and "freedom", ${ }^{21,22}$ a lack of control and lack of participation in usual activities, ${ }^{21,23,24}$ and exploratory risk taking behaviors ${ }^{25}$ may all contribute to poor adherence. Like any chronic illness, cancer has reciprocal effects on adolescent development, with effects impacting AYAs biologically, psychologically, and socially. ${ }^{25}$ Developmental factors can also influence AYAs' behavior in response to the illness; for example, AYAs' less welldeveloped abstract thinking and capacity to imagine the future, as well as associated health risk behaviors (eg, alcohol and drug use, and poor nutrition) pose additional challenges to adherence. ${ }^{25}$
Interpersonal factors can also contribute to adherence. Across chronic illnesses, poor communication and relationships, between the AYA, health care professionals (HCPs), and family, is associated with adherence challenges. ${ }^{21,26}$ In particular, overly controlling relationships with parents or $\mathrm{HCPs},{ }^{27}$ conflict between AYAs and their parents, family stress, and difficulty in delegation of treatment responsibilities can be triggers for nonadherence. ${ }^{24,28,29}$ The nature of AYAs' peer relations and social support may also contribute to adherence. . $^{21,24,30}$

Education/information regarding the illness, treatment, and side effects also appear to impact adherence in AYAs with a chronic illness. Several educational interventions have been implemented, with significant effects, although small, on adherence outcomes. ${ }^{31}$ Knowledge about treatment and illness appear essential for effective AYA health care but is not necessarily sufficient, ${ }^{21}$ with one review showing that chronic illness education alone failed to demonstrate any beneficial effects on adherence. ${ }^{32}$

Although research suggests psychological well-being plays a role in adherence for AYAs with a chronic illness, there has been little cancer-specific research. The AYA years are a time of psychological vulnerability, ${ }^{33}$ and AYAs with a chronic illness appear to be less treatment-adherent when they are experiencing high levels of stress and poor mental health. ${ }^{24,34}$ High levels of anger and low self-esteem have also been associated with greater nonadherence in adolescents. ${ }^{35}$ Depression has also been shown to be a significant risk factor for treatment nonadherence in adult and pediatric populations. ${ }^{36}$ Nonadherence in adolescents with a chronic illness may also be due to a lack of motivation. Across numerous chronic illnesses, other logistical/management factors, such as the complexity of the regimen, inability to recall instruction, poor time management, forgetfulness, treatment side effects, or inability to afford treatment, are also reported reasons for nonadherence in AYAs. ${ }^{34}$

Although AYAs with cancer face a multitude of unique challenges that are not as prevalent in other chronic illnesses, numerous common psychosocial challenges exist. Research in chronic illness therefore offers a window into understanding the AYA cancer experience. This includes translatable research in areas such as treatment adherence, as well as the role of peer interactions, therapeutic alliance and psychological impact of illness, ${ }^{37}$ and illness selfmanagement. ${ }^{38}$ Despite medical advances, cancer remains a life-threatening illness. Given the potential for nonadherence to significantly interfere with curative cancer treatment, it is critical that evidence regarding "best practice" recommen- 
dations and interventions to improve treatment adherence in AYAs be examined. Several reviews have reported that adherence-promoting interventions can improve treatment adherence in chronic illness in AYAs, ${ }^{32,39-41}$ in particular, in AYAs with diabetes, ${ }^{42}$ asthma, ${ }^{43-45}$ inflammatory bowel disease, ${ }^{46}$ and human immunodeficiency virus (HIV). ${ }^{47}$ However, less research exists that specifically addresses nonadherence among AYA cancer patients. Several unique aspects of the AYA cancer diagnosis and treatment trajectory warrant cancer-specific interventions. In particular, cancer in AYAs is fairly uncommon, ${ }^{48}$ potentially resulting in greater isolation of patients, and clinicians may have less AYA-specific skills.

To our knowledge, there are no systematic reviews available that address strategies to improve AYA adherence to cancer treatment. The primary focus of this review was therefore to synthesize the current evidence base regarding strategies that may be effective in improving treatment adherence for AYAs with cancer, in order to contribute to clinical practice and the development of evidence-based interventions in this area. In doing so, this review aimed to address two key questions:

1. What strategies have been recommended in the clinicalresearch literature to improve treatment adherence among AYAs with cancer?

2. What evidence exists to support the efficacy of adherencepromoting strategies among AYAs with cancer?

\section{Methods}

We conducted a two-staged systematic review to address our main aims. Due to the known limited research in this population, ${ }^{49}$ a narrative approach was taken to the first stage of the review, in order to synthesize current recommendations and guidelines for HCPs to improve AYAs' adherence to treatment. The second stage of the review drew together published evidence for the efficacy of adherence-promoting interventions. We followed "gold standard" systematic review procedures, using the Preferred Reporting Items for Systematic Reviews and Meta-Analyses (PRISMA) statement, to ensure the review was high quality, transparent, and comprehensive. ${ }^{50}$

The terms "adherence" and "compliance" have been used interchangeably across the literature,${ }^{51}$ with adherence differentiated by a greater level of collaboration and agreement between patient and HCP. ${ }^{10}$ Due to the common use of these terms, we decided a priori to include articles that addressed either adherence or compliance to treatment. Given current research suggesting that the term "adherence" was most appropriate,,$^{52,53}$ this term is used throughout the remainder of this review.

\section{Inclusion criteria Types of strategies}

Articles were included regardless of the type of strategy used or recommended (ie, interpersonal, intrapersonal, or a combination of both). Interpersonal-level strategies are those between individual patients and others. In our study, this included the relationship and communication between the patient and doctors, friends, and family. ${ }^{54}$ Intrapersonal strategies focus on individual factors such as health literacy, attitudes, self-efficacy, and motivation. ${ }^{55}$ Strategies that were directed toward the AYA, their parent(s), or their HCPs were included.

\section{Types of interventions}

A range of interventions have been used previously to enhance adherence in chronic illness (eg, behavioral interventions, involving strategies designed to influence behavior), informational interventions (strategies designed to educate and motivate), and family and social interventions (strategies designed to improve social support). ${ }^{56}$ Due to the paucity of intervention research addressing adherence in AYAs with cancer, all categories of interventions were eligible, and data were analyzed together. All interventions that aimed to improve adherence to any type of cancer treatment in AYAs were included (eg, medication-taking behavior, side-effect management, and nonmedical supportive care programs). ${ }^{5}$ Interventions were included irrespective of the way in which adherence was assessed (eg, observation, bioassay of serum/ urine/saliva, patient self-report, and pill counts). ${ }^{4}$

\section{Types of studies}

Studies published in peer-reviewed journals that had a focus on recommendations or strategies to improve adherence were included in the first stage of the review. Studies published in peer-reviewed journals that presented results of an intervention to improve adherence to treatment in AYAs with cancer were included in the second stage.

\section{Types of participants}

We included all articles addressing adherence in patients who were aged 10-39 years during treatment, or that specified they were relevant for adolescence and/or AYAs or tailored to the AYA developmental stage. We acknowledge that there are varying definitions of "AYA" and therefore allowed for the most broad definition to cover all relevant research. ${ }^{57}$ 
Articles that evaluated adherence in AYAs, as well as other populations (ie, pediatric, adult, geriatric) were only included if data was presented separately for each population group. Studies were eligible if the participants were receiving any form of active treatment for any type of cancer, including palliative care.

\section{Narrative synthesis of strategy recommendations}

\section{Search strategy and selection criteria}

Due to the recency of most contributions in this field, we decided to limit the search to the past 10 years, searching from 2005 to 2015 . This was supplemented by reference list and author searches. Given the growing role of Google Scholar in academic work, ${ }^{58,59}$ we utilized this search engine for the narrative stage of the review. Two grey literature databases (OpenGrey and Grey Literature Report) were also searched. The search strategy and selection criteria are summarized in Table 1.

\section{Data collection}

Two authors (EGR and KHM) searched the literature and found all eligible articles. Consensus regarding inclusion of articles was achieved by discussion.

\section{Evaluative review of interventions}

\section{Search strategy and selection criteria}

Three electronic databases were searched (MEDLINE, EMBASE, and PsychInfo), limited to human studies published in English. We limited the search to the past 10 years, searching from 2005 to 2015 . A series of searches defining the age group, disease, and outcomes were run, and the results were combined. Search terms were adapted from previous research $\mathrm{h}^{37,60}$ to ensure the most complete and accurate coverage of the literature. The search strategy and selection criteria are summarized in Table 1.

\section{Data collection}

Two authors (EGR and KHM) reviewed all abstracts and relevant full-text articles. Consensus regarding inclusion of articles was achieved by discussion. Methodological quality was assessed by two authors (EGR and KHM). Study quality was assessed using the Delphi list. The Delphi list is a set of generic core items used to assess whether the design and conduct of a randomized controlled trial is of high quality. ${ }^{61}$ The captured intervention is summarized in Table 2.

\section{Results}

\section{Study selection}

Figure 1 summarizes the search process and reasons for study exclusion. For the first stage of the systematic review, nine articles were reviewed. The interrater reliability, that is, the extent to which the two raters agreed on which articles to include, was $75 \%$ (calculated by total number of articles agreed upon, divided by total number of articles agreed upon plus total number of disagreed upon). For the second stage of the review, after deduplication, the search yielded 113 abstracts. Captured articles were screened by two authors (EGR and KHM) using the inclusion criteria described above. Three articles were deemed appropriate for full evaluation. The interrater reliability was $100 \%$. No additional articles were identified after a manual search for additional articles in relevant reviews and journals. Using the search algorithm, we were able to conduct searches with $100 \%$ sensitivity (every eligible intervention was captured by the algorithm) and $0.9 \%$ specificity (one eligible article was captured out of 113 abstracts).

Table I Summary of search algorithms

\begin{tabular}{|c|c|c|c|c|}
\hline Review & Search database & Search strategy & Data collection & Inclusion criteria \\
\hline $\begin{array}{l}\text { Narrative } \\
\text { stage }\end{array}$ & $\begin{array}{l}\text { Google Scholar, Grey } \\
\text { literature databases } \\
\text { (OpenGrey and Grey } \\
\text { Literature Report) }\end{array}$ & $\begin{array}{l}\text { Relevant publications, } \\
\text { reference lists, and author } \\
\text { searches }\end{array}$ & $\begin{array}{l}\text { - Author } \\
\text { Year of publication, country } \\
\text { Sample characteristics } \\
\text { General recommendations } \\
\text { Key statements }\end{array}$ & $\begin{array}{l}\text { Published 2005-20I5 } \\
\text { Participant age range: } 10-30 \text { yrs } \\
\text { Any active treatment } \\
\text { Any recommendation to } \\
\text { improve adherence } \\
\text { Any adherence measurement }\end{array}$ \\
\hline $\begin{array}{l}\text { Evaluative } \\
\text { stage }\end{array}$ & $\begin{array}{l}\text { MEDLINE, EMBASE, } \\
\text { and Psychlnfo }\end{array}$ & $\begin{array}{l}\text { [oncol\$ OR neoplasm OR } \\
\text { cancer OR tumor OR tumour] } \\
\text { AND [adolesc\$ OR young } \\
\text { adult OR teen OR AYA.mp OR } \\
\text { TYA.mp] AND [adherence OR } \\
\text { compliance] AND [intervention } \\
\text { OR program] AND [pilot OR } \\
\text { trial OR evaluation] }\end{array}$ & $\begin{array}{l}\text { - Author } \\
\text { Year of publication } \\
\text { Country } \\
\text { Sample/intervention } \\
\text { characteristics } \\
\text { Study methods } \\
\text { Intervention outcomes } \\
\text { Methodological quality }\end{array}$ & $\begin{array}{l}\text { Published 2005-20I5 } \\
\text { Participant age range: 10-30 yrs } \\
\text { Any active treatment } \\
\text { Any type of intervention to } \\
\text { improve adherence } \\
\text { Any adherence measurement }\end{array}$ \\
\hline
\end{tabular}




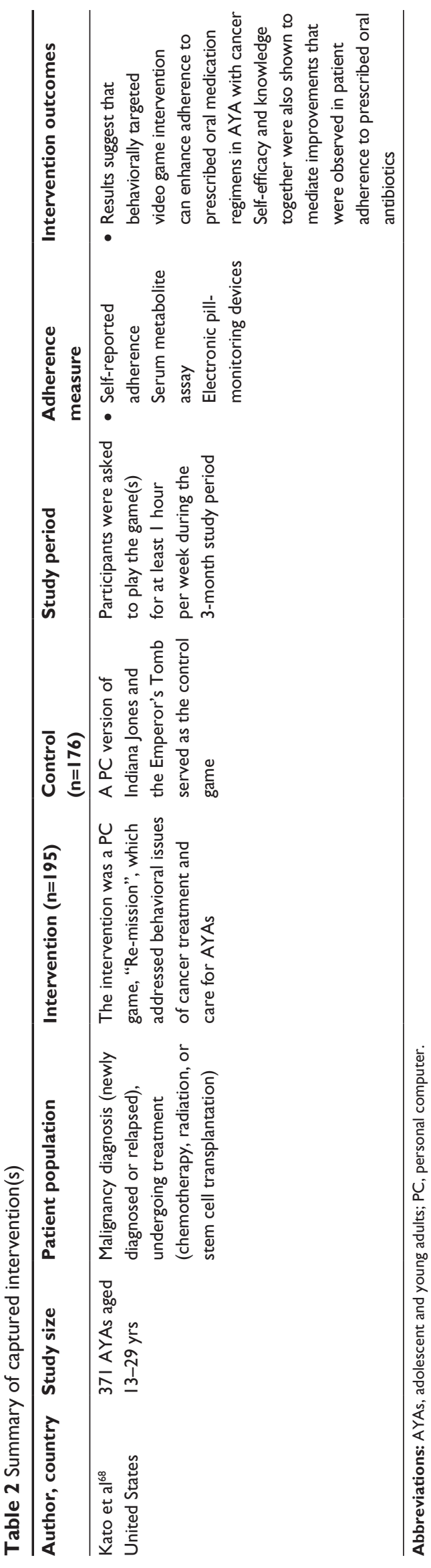

\section{Research question I: what types of strategies have been recommended in the clinical-research literature to improve treatment adherence among AYAs with cancer? \\ Description of articles}

Nine articles that explored recommendations for treatment adherence among AYAs with cancer were found that met the inclusion criteria. Five articles were narrative reviews, ${ }^{4,5,9,62,63}$ two reported studies involving semistructured interviews, ${ }^{64,65}$ one was an analysis of qualitative focus group data, ${ }^{66}$ and one was a proposed algorithm for adherence risk assessment. ${ }^{19}$ Five articles addressed cancer patients in general, 4,5,9,19,62 three assessed acute lymphoblastic leukemia patients specifically, ${ }^{64-66}$ and one addressed patients with any hematological disorder. ${ }^{63}$ The articles are summarized in Table 3.

In terms of participants' ages at the time of treatment, five articles discussed AYAs, ${ }^{4,5,19,63,66}$ two articles discussed adolescents only, ${ }^{9,62}$ one article discussed children, adolescents, and adults, ${ }^{64}$ and one article discussed children and adolescents. ${ }^{65}$

\section{Findings}

The nine reviewed articles described a range of strategies to be implemented by the AYA, parent, and/or HCP. The identified strategies targeted a range of underlying factors, and we categorized these strategies into five domains that affect adherence: developmental, communication, educational, psychological well-being, and logistical/management difficulties. Articles that discussed strategies taking a developmental approach were the most prevalent $(n=9), 4,9,11,19,62-66$ while strategies relating to communication were identified in six articles. ${ }^{911,19,62,65,66}$ Table 4 provides an overview of the strategies recommended to address each underlying factor, for AYA patients, parents, and HCPs.

\section{Developmental strategies}

All nine articles highlighted the role that developmental stage might play in impeding treatment adherence among AYAs with cancer. Development-focused recommendations appeared to take two forms. Firstly, some strategies focused on how parents and HCPs can present, and discuss treatment information in a developmentally appropriate manner. Taking the developmental stage and maturity of the patient into consideration when developing treatment plans may assist in improving adherence outcomes. ${ }^{19,64-66}$ In particular, providing AYAs with a greater sense of control and allowing them to maintain independence throughout treatment may 

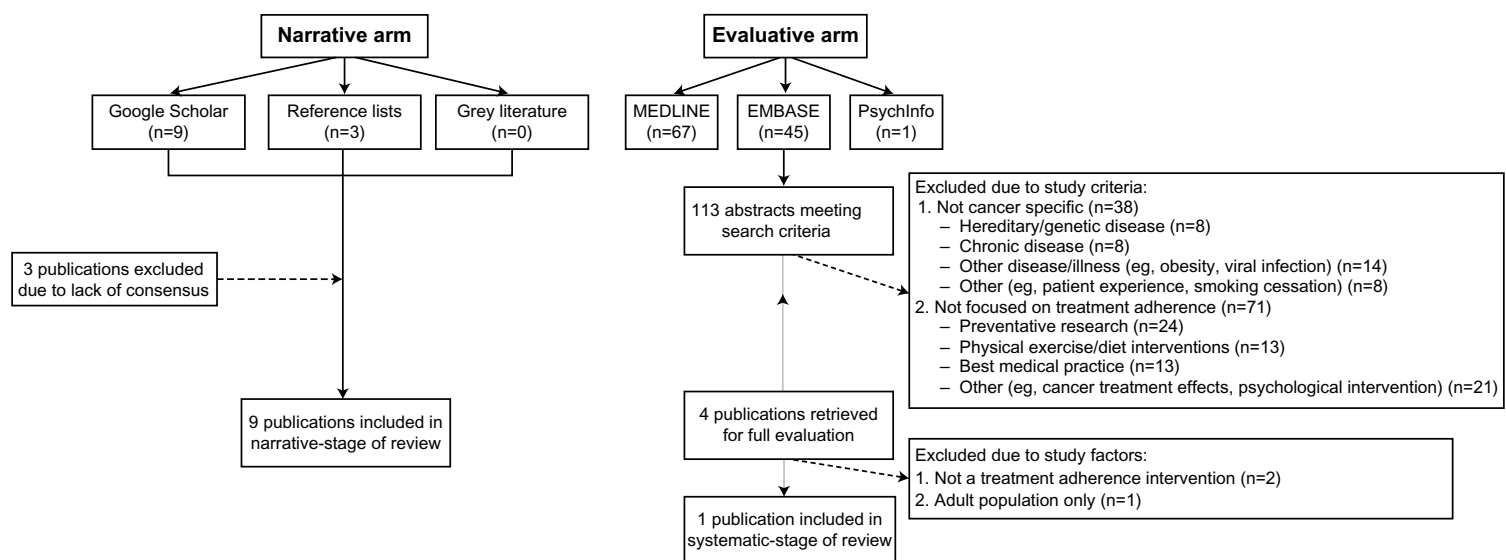

Figure I Reasons for exclusion of publications resulting from the database search.

improve adherence. ${ }^{62,65}$ Secondly, other strategies focused on how AYA milestones, such as school formals, graduations, and 18th birthdays, should be addressed through collaboration of the AYA, their parents, and HCPs. ${ }^{11}$ Recommendations were that active discussions between the three parties should focus on how the AYA can continue to meet important milestones whilst meeting an adequate level of treatment adherence; ${ }^{63,66}$ by acknowledging the developmental importance of such social events and allowing for flexibility in the treatment schedule, these strategies promote adherence in the context of the unique developmental issues in AYAs. ${ }^{19,66}$

\section{Communication-related strategies}

Six articles emphasized the important role that communication has in treatment adherence among AYAs with cancer, 9,11,19,62,63,65 highlighting that AYAs, parents, and HCPs need to work together throughout the treatment process to ensure good adherence.

Articles recommended strategies in which the HCP tailors communication style to the individual patient, with the aim of achieving open and trustworthy communication. ${ }^{9,67}$ Additionally, positive family relationships and good communication between AYAs and parents is also important for adherence. 4,11,63 Agreement with parents about treatment, medication, and the doctor's instructions is especially important. ${ }^{62}$ Role delineation about medication administration also needs to be clarified between AYAs and their parents in the early stages of treatment. ${ }^{4,9,65}$

\section{Educational and informational strategies}

The role of education and information about the illness and treatment was emphasized in three articles. ${ }^{11,63,65}$ Adherence may be improved when the patient understands the treatment procedures, and the effects of treatment and medications. ${ }^{63}$
The literature identified that HCPs need to provide AYAs with knowledge about the disease, treatment, and future health outcomes, and provide them with confidence in the possibility of their recovery. ${ }^{9}$ Articles highlighted the importance of AYAs understanding the life-threatening nature of their disease. ${ }^{19,65}$ Personal health beliefs and locus of control may also influence adherence. ${ }^{4,11}$ The most important strategy discussed was providing education about medication, the therapeutic effects of the lifesaving treatment, and the importance of taking medication when advised. ${ }^{11,65}$ Articles also suggested that HCPs and parents need to be responsible for teaching AYAs basic adherence strategies, and encouraged strategies such as the use of reminder tools and instilling medication routines. ${ }^{11,65}$ Further recommendations are that AYAs should also seek information by raising any concerns or questions with their clinician; in response, HCPs should aim to provide a safe and nonjudgmental environment for the AYA's concerns to be raised, and as well remain openminded and willing to discuss or offer alternative treatment options when appropriate. ${ }^{62}$ Parents' knowledge about cancer and its treatment is also associated with adherence. ${ }^{65}$ Articles also focused on HCPs, arguing that they are responsible for ensuring they are up to date with current chronic illness adherence strategies for AYAs. ${ }^{9,11}$

\section{Psychological strategies}

Several articles discussed the importance of psychological well-being in treatment adherence among AYAs with cancer. ${ }^{4,19,63}$ Nonadherence can be seen as a sign of distress. ${ }^{4}$ Specifically, psychological factors such as parental depression, ${ }^{62}$ anxiety, ${ }^{63}$ and self-esteem ${ }^{4}$ were identified as potentially affecting AYAs' treatment adherence. Articles argued that HCPs and parents must acknowledge the potential for psychological distress, and minimize the risk by providing 


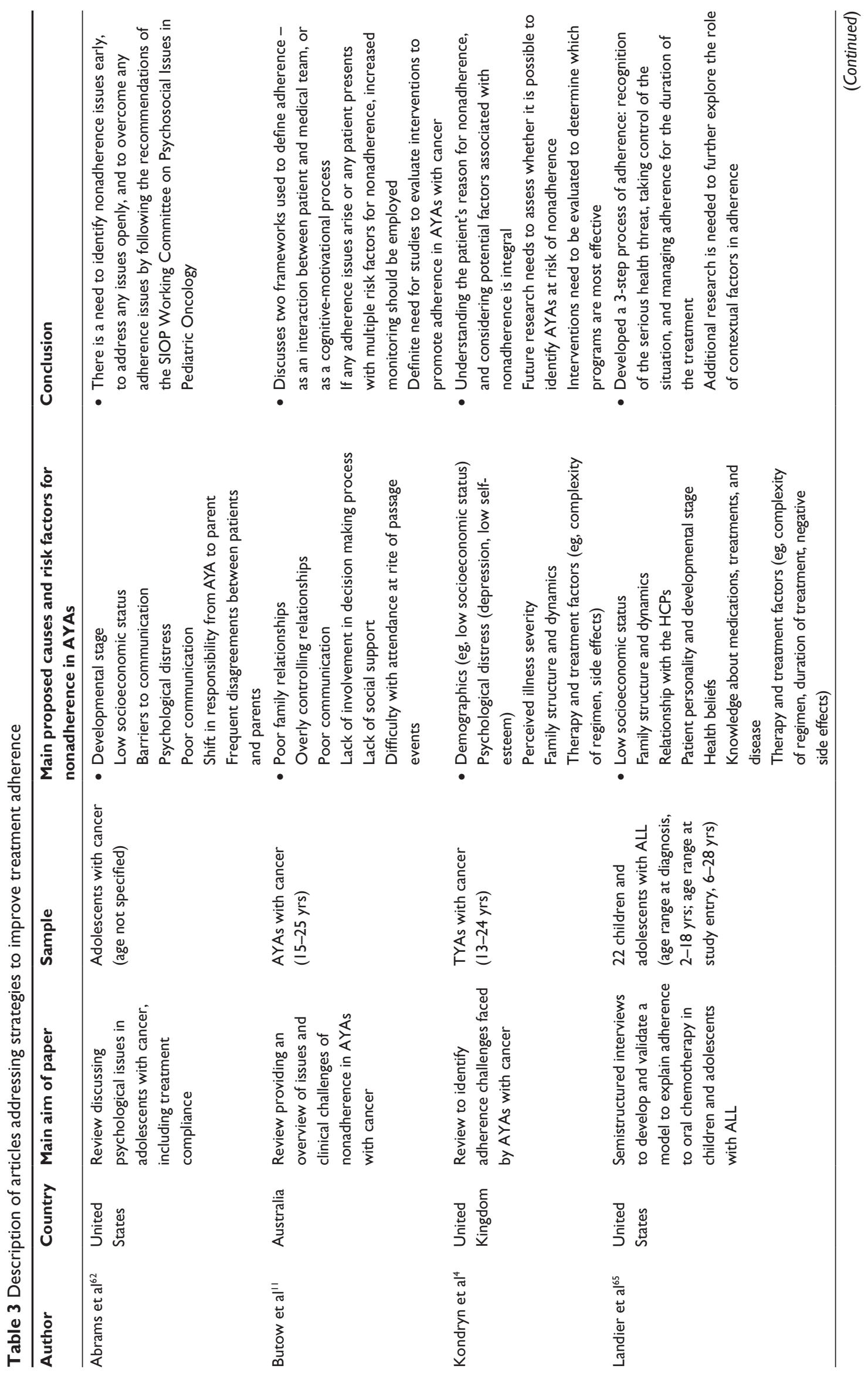




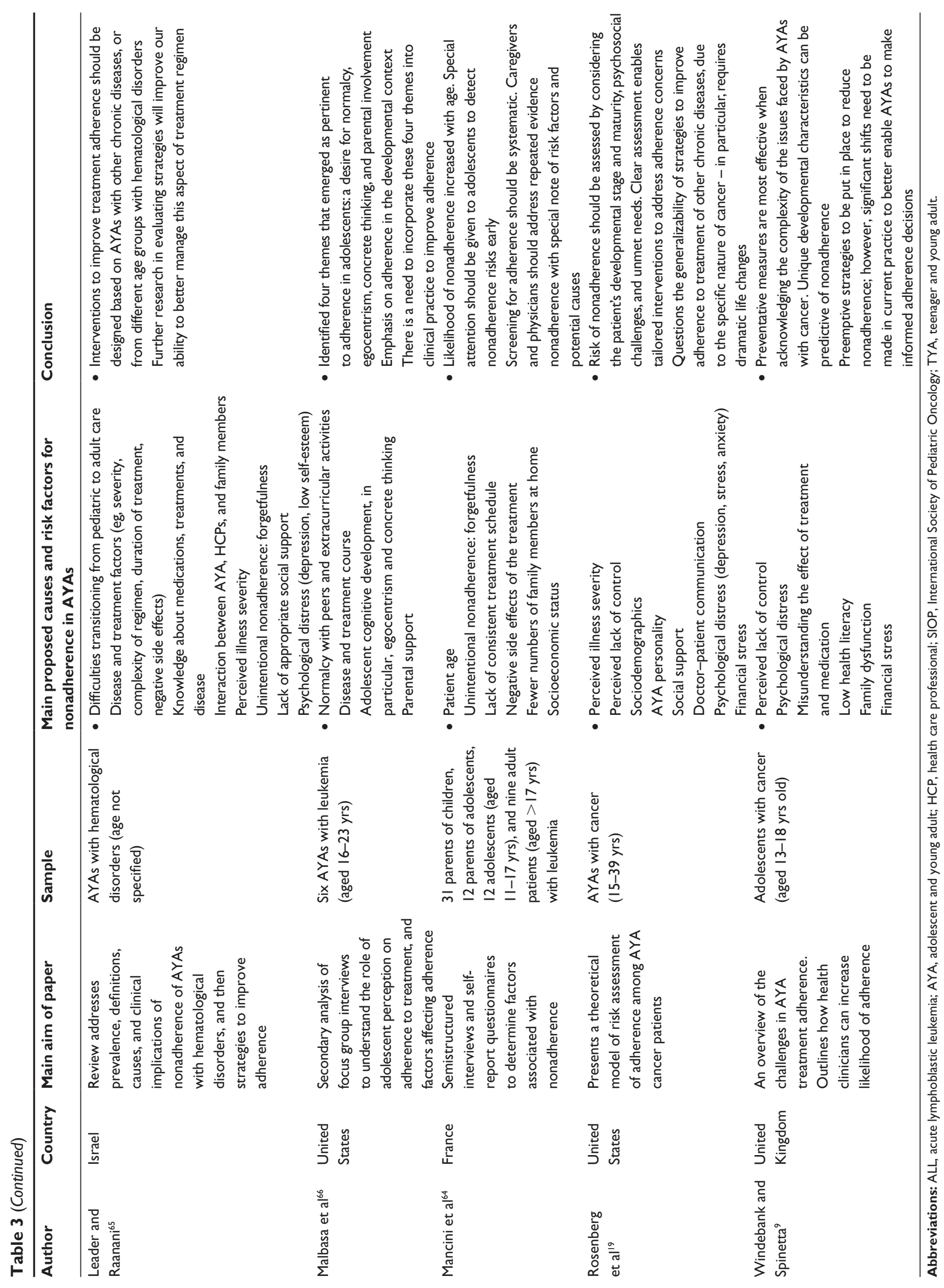




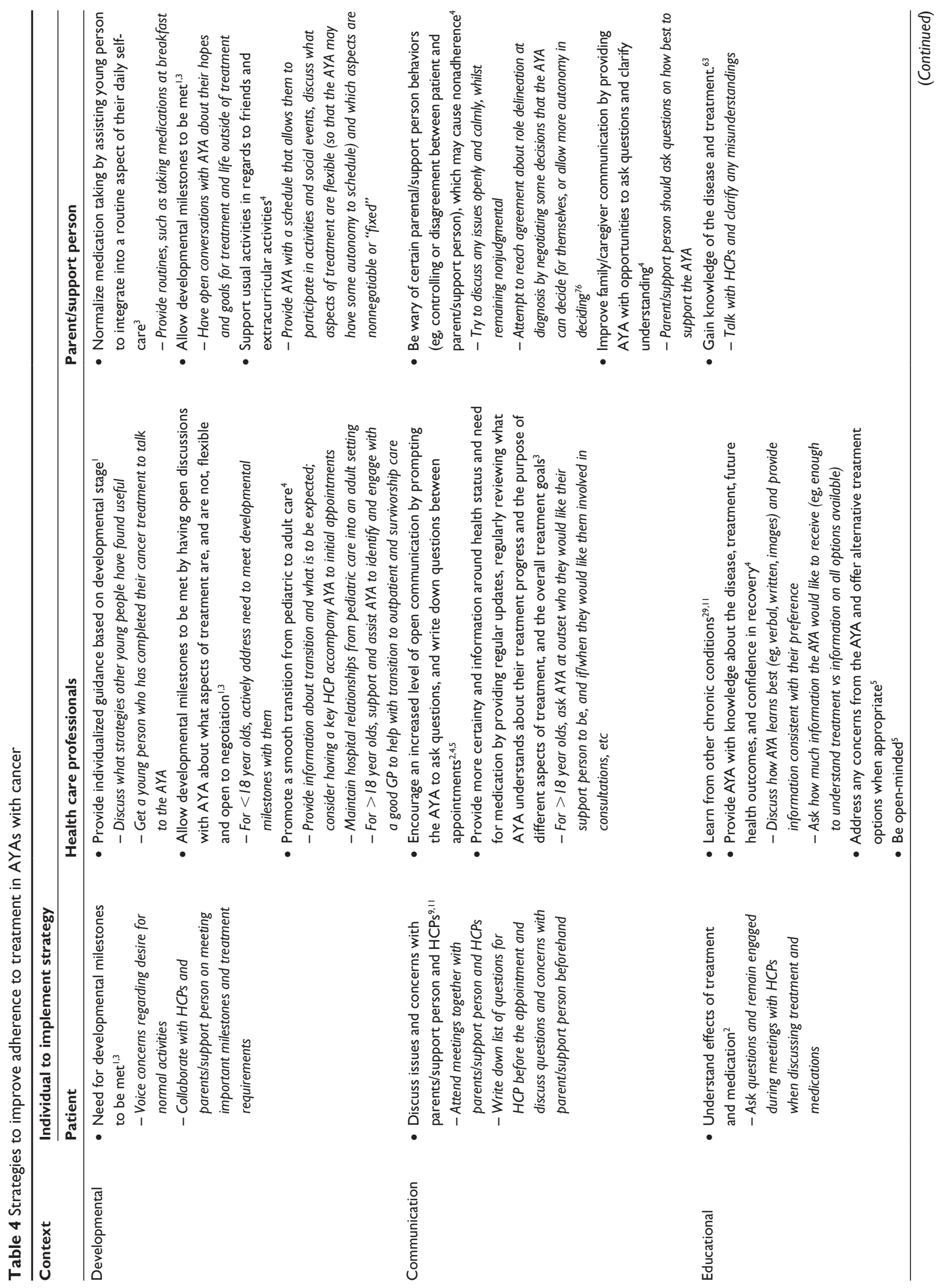




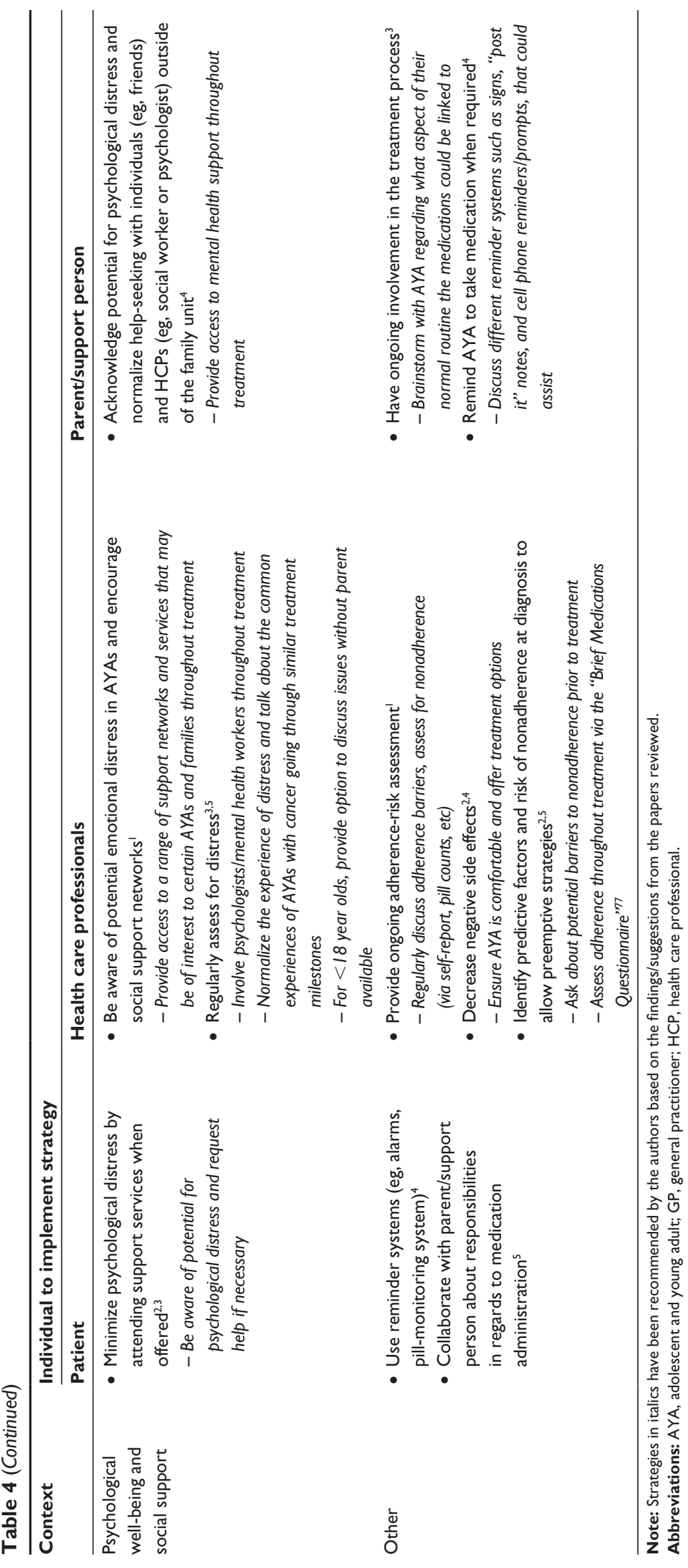


the AYA with access to further psychosocial support services. ${ }^{4,62}$ By maintaining a positive attitude, being aware of their own psychological states, and accepting support when offered, AYAs can also reduce the risk of any psychological distress and counter any potential adherence issues. ${ }^{65}$

\section{Other strategies}

Finally, other factors regarding the logistics and management of treatment, complex regimens, and treatment side effects were also highlighted as potentially impairing AYAs' capacity to adhere to treatment. ${ }^{4,5,19,63,64,66}$ Articles argued that HCPs need to tailor treatment plans to meet individual patient needs ${ }^{66}$ and that parents and AYAs should assess what treatment routines work best and establish a treatment management schedule that both parties agree on. ${ }^{4,65}$

\section{Research question 2: what evidence exists to support the efficacy of adherence- promoting strategies among AYAs with cancer? \\ Description of intervention(s)}

Only one intervention met criteria for inclusion in this systematic review. It assessed the effectiveness of a computer game intervention for improving treatment adherence and other behavioral outcomes for AYAs with cancer. The game addressed issues including common treatment-related adverse effects (eg, bacterial infection and nausea), and positive self-care behaviors (eg, taking oral chemotherapy and practicing good mouth care). The intervention translated behavioral objectives into the game, based on principles of the self-regulation model of health and illness, social cognitive theory, and learning theory.

\section{Methodological quality of intervention(s)}

Two investigators (EGR and CEW) evaluated the intervention: the Delphi score was 7 (from a possible range of 0-9), indicating moderate-high methodological quality (interrater reliability of the Delphi scores was 100\%). However, Delphi items regarding concealment of treatment allocation and blinding of patients were not met.

\section{Intervention outcomes}

Results showed that although both groups of AYAs (those who participated in the game and those who did not) described themselves as highly treatment-adherent across time points, there was a significant $16 \%$ increase in antibiotic adherence for the intervention group. Oral chemotherapy adherence also remained significantly higher in the intervention group than in the control group. Increases in cancer-related knowledge and cancer-specific self-efficacy were also reported, with these changes mediating improvements observed in AYA adherence to oral antibiotics. Less than 30\% of AYAs fully adhered to the intervention requirements; however results still indicated significant benefits on medication adherence, despite suboptimal game play (less than $50 \%$ of the requested game time).

\section{Discussion}

Research in AYA adherence to cancer treatment is lacking. With recent research focusing on the barriers affecting adherence in AYAs with cancer, published recommendations have increasingly focused on adherence-promoting strategies for these patients, parents, and HCPs. The present review synthesized recommendations for improving cancer treatment adherence within AYA populations, and summarized the evidence for adherence-promoting interventions. While some recommendations were extracted from the existing literature, the review highlights the dearth of fully tested strategies available to improve adherence outcomes in AYAs affected by cancer, with the description of only one evidence-based intervention.

Several commonalities in barriers to adherence and strategies to improve nonadherence were found across the reviewed articles and were categorized according to five domains: developmental, communication, education, psychological well-being, and logistical/management difficulties. Key to the present findings was the acknowledgment of the AYA developmental stage and the unique challenges it presents. Strategies supporting AYAs' need for "normalcy" were most prevalent. ${ }^{66}$ Open and nonjudgmental communication between HCPs, patient, and family was also strongly recommended to improve adherence. Providing the AYA and parent with information about the disease, treatment procedures, and medications is also important. HCPs also need to be aware of strategies to improve adherence in AYAs with chronic illness as a starting point for addressing adherence in AYAs with cancer. Minimizing psychological distress, providing support services, and improving social support networks may also lead to improved adherence. Other strategies, such as reducing treatment side effects, identifying patients at risk for adherence problems at diagnosis, and reminder systems for commonly cited forgetfulness, may also lead to improved adherence.

Highlighting the paucity of adherence interventions, this review identified only one study that systematically evaluated the role of an intervention on adherence behaviors. ${ }^{68}$ 
The study showed a behaviorally targeted computer game could improve adherence to oral antibiotics and chemotherapy. Cancer-related self-efficacy and knowledge was also shown to mediate adherence. This intervention took an educational approach and focused on the AYA patient developing specific knowledge about their cancer, the role of treatment and common side effects, and the importance of adherence, with no involvement of the parents or HCPs in the intervention. Given the reviewed evidence indicating the importance of communication, support, and interactions with parents and HCPs, future interventions might build on this study. Combining the efficacious education strategies for AYAs used in this intervention, with other strategies (eg, communication-focused strategies) may achieve even stronger effect.

\section{AYA adherence: implications for future interventions}

Even with a growing evidence base documenting the challenges AYAs face with adherence to cancer treatment, few studies provide evidence-based advice surrounding strategies to improve adherence, and only one rigorously tested intervention has been described in the literature in the last 10 years. The results of this review also indicate that the majority of the recommended strategies in this area are interpersonal, focusing on communication between patients and HCPs, rather than focusing on strategies that AYAs can implement themselves. Future interventions work may draw on the broader chronic illness literature to implement more communications-focused strategies among AYAs with cancer.

Strategies recommended to improve adherence were for implementation by the patient, parent, or a HCP. Adherence, by definition, involves the patient actively working to maintain their health. ${ }^{21}$ In understanding nonadherence, the interaction between patients and others, and the patients cognitive-motivational processes both need to be taken into consideration. ${ }^{5}$ To better manage issues of nonadherence, research focusing on both intrapersonal strategies and the cognitive-motivational processes of AYAs is necessary. In addition to strategies for parents and HCPs, interventions for AYAs that address coping strategies, health beliefs, understanding and managing treatment, and motivation may improve adherence further.

Although AYA adherence to cancer treatment requires further exploration, it may be beneficial to build upon the literature among AYAs with other chronic illnesses and cancer in other age groups, where similar adherence challenges exist. ${ }^{4,11,63}$ Research has highlighted the similar psychosocial challenges that face AYAs living with chronic illnesses across various conditions; ${ }^{37,69}$ existing adherence-promoting interventions for AYAs with other chronic illnesses may be used to develop targeted interventions for AYAs with cancer. Many strategies to improve adherence recommended for AYAs with cancer correspond to strategies for AYAs with a chronic illness, including balancing AYA life priorities and the treatment regimen, decreasing side effects, ${ }^{30}$ and using reminders to reduce forgetfulness. ${ }^{34}$

The World Health Organization has argued that increasing the effectiveness of adherence interventions may have a greater impact than improvement in specific medical treatments for chronic illnesses. ${ }^{70}$ One review that assessed the effects of 182 interventions to enhance chronic illness patients' medication adherence, however, found effects were somewhat inconsistent from study to study. ${ }^{71}$ The authors concluded that the current methods for improving adherence in chronic illness are overly complex (reducing the ability to replicate studies) and not very effective. ${ }^{71}$ However, another review found adherence-promoting interventions were effective, although effects had limited longevity. ${ }^{72}$ Across chronic illnesses in children and adolescents, behavioral and multicomponent interventions appear to be most effective at improving adherence, with medium effect sizes. ${ }^{31}$ An education program for parents of childhood cancer patients also had a positive outcome on adherence, with a significant decrease in treatment refusal. ${ }^{73}$ Future interventions should also consider being guided by the Adolescent Resilience Model, specifically developed as a model for understanding the process of resilience and quality of life outcomes in adolescents with cancer. ${ }^{74,75}$ Motivational Enhancement Therapy, which focuses on trying to understand an adolescent's view, rather than coercing them to change their behaviors, and may also be of use in improving adherence in children and adolescents. ${ }^{41}$

\section{Limitations}

To our knowledge, this was the first systematic review to synthesize the evidence base for adherence-promoting strategies among AYAs with cancer. This addresses a significant gap in the literature and outlines individual strategies researchers and clinicians may implement, highlighting important methodological "next steps" for interventions research in this field. The results of this review should be interpreted in reference to a number of limitations, however. Although a strength of this paper is that it broadly covers the spectrum of adherences issues in AYAs, this is also a limitation, in that specific subgroups of AYAs, such as those aged between 10 and 15 years old, were not commonly addressed 
throughout this paper. This is also a limitation in the literature, due to minimal research differentiating subgroups within the AYA population. ${ }^{60}$ As only articles that separately analyzed a subset of AYA participants were included, an additional limitation of our paper is the potential of having missed data from the broader adult oncology literature. As the aim of the study was to review strategies to improve current treatment adherence in AYAs, only articles published since 2005 were included due to improvements in cancer treatment. Although this methodological decision was to identify the strategies based on current medical treatment, it may have resulted in exclusion of appropriate, older, articles. Another limitation was the single intervention identified in the review. Although this reflects the paucity of literature in this field, the examination of only one intervention limited our ability to examine adherence strategies across studies and draw conclusions.

\section{Conclusion and future directions}

With the gap widening between potential and actual patient health, there is an increasing need to address malleable factors that improve AYA health outcomes, such as treatment adherence. This review found that effective treatment adherence is best promoted through a collaborative approach involving patients, parents, and HCPs. Emphasis was placed on improving communication, addressing developmental concerns for the AYA, and reducing patient distress. Based on the findings of this review, the authors recommend that more effort be devoted to developing interventions with specific strategies that aim to improve adherence in AYAs. Development of targeted interventions and adherence strategies could be adapted from those identified in research of chronic illness in AYAs. Future research should also consider the wide variability of experiences within the AYA stage, such as the role of parents as opposed to spouses or of attendance at school versus work, and the relationship between these individual variables on adherence. Regardless, the strategies adopted should be individually tailored for each patient, given their unique developmental needs and individual risk or resilience factors. By addressing individual barriers to care, more appropriate strategies can be implemented. Improving adherence in AYAs with cancer has the potential to ultimately improve health outcomes, reduce patient distress, provide more sound clinical trials results, and lead to a more cost-effective health care system.

\section{Acknowledgments}

The authors wish to acknowledge the support of Associate Professor Richard Cohn, Head of Clinical Oncology, Sydney Children's Hospital, and Ms Brittany McGill (Clinical Psychologist,
Behavioural Sciences Unit). The Behavioural Sciences Unit is supported by the Kids with Cancer Foundation.

\section{Disclosure}

Claire E Wakefield is supported by a Career Development Fellowship from the National Health and Medical Research Council of Australia (grant number APP1067501) and an Early Career Development Fellowship from the Cancer Institute of NSW (grant number 11/ECF/3-43). Ursula M Sansom-Daly is supported by an Early Career Fellowship from the Cancer Institute of NSW (grant number 14/ECF/1-11). The authors report no other conflicts of interest in this work.

\section{References}

1. Barr RD. Adolescents, young adults, and cancer - the international challenge. Cancer. 2011;117(10 Suppl):2245-2249.

2. Bleyer A. Young adult oncology: the patients and their survival challenges. CA Cancer J Clin. 2007;57(4):242-255.

3. Alston RD, Rowan S, Eden TO, Moran A, Birch JM. Cancer incidence patterns by region and socioeconomic deprivation in teenagers and young adults in England. Br J Cancer. 2007;96(11):1760-1766.

4. Kondryn HJ, Edmondson CL, Hill J, Eden TO. Treatment nonadherence in teenage and young adult patients with cancer. Lancet Oncol. 2011;12(1):100-108.

5. Luckett T, Butow PN, King MT, et al. A review and recommendations for optimal outcome measures of anxiety, depression and general distress in studies evaluating psychosocial interventions for Englishspeaking adults with heterogeneous cancer diagnoses. Support Care Cancer. 2010;18(10):1241-1262.

6. Ruddy K, Mayer E, Partridge A. Patient adherence and persistence with oral anticancer treatment. CA Cancer J Clin. 2009;59(1):56-66.

7. Bleyer WA. Cancer in older adolescents and young adults: epidemiology, diagnosis, treatment, survival, and importance of clinical trials. Med Pediatr Oncol. 2002;38(1):1-10.

8. Tebbi CK. Treatment compliance in childhood and adolescence. Cancer. 1993;71(S10):3441-3449.

9. Windebank KP, Spinetta JJ. Do as I say or die: compliance in adolescents with cancer. Pediatr Blood Cancer. 2008;50(5 Suppl):1099-1100.

10. World Health Organization. Adherence to Long-Term Therapies: Evidence for Action. Geneva: World Health Organization; 2003.

11. Butow P, Palmer S, Pai A, Goodenough B, Luckett T, King M. Review of adherence-related issues in adolescents and young adults with cancer. J Clin Oncol. 2010;28(32):4800-4809.

12. Brown MT, Bussell JK. Medication adherence: WHO cares? Mayo Clin Proc. 2011;86(4):304-314.

13. Partridge AH, Avorn J, Wang PS, Winer EP. Adherence to therapy with oral antineoplastic agents. J Natl Cancer Inst. 2002;94(9):652-661.

14. Peeters M, Koren G, Jakubovicz D, Zipursky A. Physician compliance and relapse rates of acute lymphoblastic leukemia in children. Clin Pharmacol Ther. 1988;43(3):228-232.

15. Lilleyman JS, Lennard L. Non-compliance with oral chemotherapy in childhood leukaemia. BMJ. 1996;313(7067):1219-1220.

16. Hershman DL, Shao T, Kushi LH, et al. Early discontinuation and nonadherence to adjuvant hormonal therapy are associated with increased mortality in women with breast cancer. Breast Cancer Res Treat. 2011;126(2):529-537.

17. Eliasson L, Jackson C, Brady R, Kalthoff J. Improving adherence to oral cancer treatments. Hospital Pharmacy Europe. 2012;65:43-45.

18. Roter DL, Hall JA, Merisca R, Nordstrom B, Cretin D, Svarstad B. Effectiveness of interventions to improve patient compliance: a meta-analysis. Med Care. 1998;36(8):1138-1161. 
19. Rosenberg AR, Macpherson CF, Kroon L, Johnson R. Rethinking adherence: A proposal for a new approach to risk assessment. J Adolesc Young Adult Oncol. 2013;2(2):83-86.

20. Adolescent and Young Adult Oncology Progress Review Group. Closing the Gap: Research and Care Imperatives for Adolescents and Young Adults with Cancer. Bethesda, MD: National Cancer Institute; 2006.

21. Kyngäs HA, Kroll T, Duffy ME. Compliance in adolescents with chronic diseases: a review. J Adolesc Health. 2000;26(6):379-388.

22. Dziuban EJ, Saab-Abazeed L, Chaudhry SR, Streetman DS, Nasr SZ. Identifying barriers to treatment adherence and related attitudinal patterns in adolescents with cystic fibrosis. Pediatr Pulmonol. 2010;45(5): 450-458.

23. Sawyer SM, Aroni RA. Self-management in adolescents with chronic illness. What does it mean and how can it be achieved. Med J Aust. 2005;183(8):405-409.

24. Yeo M, Sawyer S. ABC of adolescence: Chronic illness and disability. BMJ. 2005;330(7493):721-723.

25. Suris J, Michaud P, Viner R. The adolescent with a chronic condition. Part I: developmental issues. Arch Dis Child. 2004;89(10):938-942.

26. Bullington P, Pawola L, Walker R, Valenta A, Briars L, John E. Identification of medication non-adherence factors in adolescent transplant patients: the patient's viewpoint. Pediatr Transplant. 2007;11(8): 914-921.

27. Kyngäs H, Hentinen M, Barlow JH. Adolescents' perceptions of physicians, nurses, parents and friends: help or hindrance in compliance with diabetes self-care? J Adv Nurs. 1998;27(4):760-769.

28. Naimi DR, Freedman TG, Ginsburg KR, Bogen D, Rand CS, Apter AJ. Adolescents and asthma: why bother with our meds? J Allergy Clin Immunol. 2009;123(6):1335-1341.

29. Gray WN, Denson LA, Baldassano RN, Hommel KA. Treatment adherence in adolescents with inflammatory bowel disease: the collective impact of barriers to adherence and anxiety/depressive symptoms. J Pediatr Psychol. 2012;37(3):282-291.

30. Taddeo D, Egedy M, Frappier JY. Adherence to treatment in adolescents. Paediatr Child Health. 2008;13(1):19-24.

31. Kahana S, Drotar D, Frazier T. Meta-analysis of psychological interventions to promote adherence to treatment in pediatric chronic health conditions. J Pediatr Psychol. 2008;33(6):590-611.

32. Dean AJ, Walters J, Hall A. A systematic review of interventions to enhance medication adherence in children and adolescents with chronic illness. Arch Dis Child. 2010;95(9):717-723.

33. Sansom-Daly UM, Wakefield CE. Distress and adjustment among adolescents and young adults with cancer: An empirical and conceptual review. Transl Pediatr. 2013;2(4):167-197.

34. Hanghøj S, Boisen KA. Self-reported barriers to medication adherence among chronically ill adolescents: a systematic review. $J$ Adolesc Health. 2014;54(2):121-138.

35. Penkower L, Dew MA, Ellis D, Sereika SM, Kitutu JM, Shapiro R. Psychological distress and adherence to the medical regimen among adolescent renal transplant recipients. Am J Transplant. 2003;3(11): $1418-1425$

36. DiMatteo MR, Lepper HS, Croghan TW. Depression is a risk factor for noncompliance with medical treatment: meta-analysis of the effects of anxiety and depression on patient adherence. Arch Intern Med. 2000;160(14):2101-2107.

37. Sansom-Daly UM, Peate M, Wakefield CE, Bryant RA, Cohn RJ. A systematic review of psychological interventions for adolescents and young adults living with chronic illness. Health Psychol. 2012;31(3): 380-393.

38. Lawn S. What can cancer care learn from the chronic condition management field? Presented at: Flinders Centre for Innovation in Cancer Survivorship Conference; February 1-3; 2013; Adelaide, South Australia.

39. Graves MM, Roberts MC, Rapoff M, Boyer A. The efficacy of adherence interventions for chronically ill children: a meta-analytic review. J Pediatr Psychol. 2010;35(4):368-382.
40. Pai AL, McGrady M. Systematic review and meta-analysis of psychological interventions to promote treatment adherence in children, adolescents, and young adults with chronic illness. J Pediatr Psychol. 2014;39(8):918-931.

41. Lask B. Motivating children and adolescents to improve adherence. J Pediatr. 2003;143(4):430-433.

42. Ellis DA, Naar-King S, Chen X, Moltz K, Cunningham PB, IdalskiCarcone A. Multisystemic therapy compared to telephone support for youth with poorly controlled diabetes: findings from a randomized controlled trial. Ann Behav Med. 2012;44(2):207-215.

43. Burkhart PV, Rayens MK, Oakley MG, Abshire DA, Zhang M. Testing an intervention to promote children's adherence to asthma self-management. J Nurs Scholarsh. 2007;39(2):133-140.

44. Duncan CL, Hogan MB, Tien KJ, et al. Efficacy of a parent-youth teamwork intervention to promote adherence in pediatric asthma. J Pediatr Psychol. 2013;38(6):617-628.

45. Otsuki M, Eakin MN, Rand CS, et al. Adherence feedback to improve asthma outcomes among inner-city children: a randomized trial. Pediatrics. 2009;124(6):1513-1521.

46. Hommel KA, Hente EA, Odell S, et al. Evaluation of a group-based behavioral intervention to promote adherence in adolescents with inflammatory bowel disease. Eur J Gastroenterol Hepatol. 2012;24(1): 64-69.

47. Letourneau EJ, Ellis DA, Naar-King S, Chapman JE, Cunningham PB, Fowler S. Multisystemic therapy for poorly adherent youth with HIV: results from a pilot randomized controlled trial. AIDS Care. 2013;25(4): 507-514.

48. Bleyer A, O'Leary M, Barr R, Ries LAG, editors. Cancer Epidemiology in Older Adolescents and Young Adults 15 to 29 Years of Age, Including SEER Incidence and Survival: 1975-2000. Bethesda, MD: National Cancer Institute; 2006.

49. Thomas DM, Seymour JF, O'Brien T, Sawyer SM, Ashley DM. Adolescent and young adult cancer: a revolution in evolution? Intern Med J. 2006;36(5):302-307.

50. Moher D, Liberati A, Tetzlaff J, Altman DG; PRISMA Group. Preferred reporting items for systematic reviews and meta-analyses: the PRISMA statement. Ann Intern Med. 2009;151(4):264-269.

51. Horne R, Weinman J, Barber N, et al. Concordance, Adherence and Compliance in Medicine Taking. London: National Co-ordinating Centre for NHS Service Delivery and Organisation (NCCSDO); 2005.

52. Tilson HH. Adherence or compliance? Changes in terminology. Ann Pharmacother. 2004;38(1):161-162.

53. Vermeire E, Hearnshaw H, Van Royen P, Denekens J. Patient adherence to treatment: three decades of research. A comprehensive review. J Clin Pharm Ther. 2001;26(5):331-342.

54. Bosworth HB, Oddone EZ, Weinberger M, editors. Patient Treatment Adherence: Concepts, Interventions, and Measurement. New York, NY: Psychology Press; 2008.

55. Glanz K, Lewis FM, Rimer BK. Health Behavior and Health Education: Theory, Research, and Practice. San Francisco, CA: Jossey-Bass; 1990.

56. Kripalani S, Yao X, Haynes RB. Interventions to enhance medication adherence in chronic medical conditions: a systematic review. Arch Intern Med. 2007;167(6):540-550.

57. National Cancer Institute. A Snapshot of Adolescent and Young Adult Cancers. Bethesda, MD: National Cancer Institute; 2014

58. Jacsó P. Google Scholar: the pros and the cons. Online Inform Rev. 2005;29(2):208-214.

59. Falagas ME, Pitsouni EI, Malietzis GA, Pappas G. Comparison of PubMed, Scopus, Web of Science, and Google Scholar: strengths and weaknesses. FASEB J. 2008;22(2):338-342.

60. Wakefield CE, Patterson P, McDonald FE, Wilson HL, Davis E, Sansom-Daly UM. Assessment of psychosocial outcomes in adolescents and young adults with cancer: a systematic review of available instruments. Clin Oncol Adolesc Young Adults. 2013;2013(3): $13-27$. 
61. Verhagen AP, de Vet HC, de Bie RA, et al. The Delphi list: a criteria list for quality assessment of randomized clinical trials for conducting systematic reviews developed by Delphi consensus. J Clin Epidemiol. 1998;51(12):1235-1241.

62. Abrams AN, Hazen EP, Penson RT. Psychosocial issues in adolescents with cancer. Cancer Treat Rev. 2007;33(7):622-630.

63. Leader A, Raanani P. Adherence-related issues in adolescents and young adults with hematological disorders. Acta Haematol. 2014;132(3-4): 348-362.

64. Mancini J, Simeoni MC, Parola N, et al. Adherence to leukemia maintenance therapy: a comparative study among children, adolescents, and adults. Pediatr Hematol Oncol. 2012;29(5):428-439.

65. Landier W, Hughes CB, Calvillo ER, et al. A grounded theory of the process of adherence to oral chemotherapy in Hispanic and caucasian children and adolescents with acute lymphoblastic leukemia. J Pediatr Oncol Nurs. 2011;28(4):203-223.

66. Malbasa T, Kodish E, Santacroce SJ. Adolescent adherence to ora therapy for leukemia: a focus group study. J Pediatr Oncol Nurs. 2007;24(3):139-151.

67. Dunn LB, Cooper BA, Neuhaus J, et al. Identification of distinct depressive symptom trajectories in women following surgery for breast cancer. Health Psychol. 2011;30(6):683-692.

68. Kato PM, Cole SW, Bradlyn AS, Pollock BH. A video game improves behavioral outcomes in adolescents and young adults with cancer: a randomized trial. Pediatrics. 2008;122(2):e305-e317.

69. Eiser C. What can we learn from other illnesses? J Cyst Fibros. 2003; 2(1):58-60.
70. Haynes RB, McDonald H, Garg AX, Montague P. Interventions for helping patients to follow prescriptions for medications. Cochrane Database Syst Rev. 2002;2:CD000011.

71. Nieuwlaat R, Wilczynski N, Navarro T, et al. Interventions for enhancing medication adherence. Cochrane Database Syst Rev. 2014;11:CD000011.

72. Cortina S, Somers M, Rohan JM, Drotar D. Clinical effectiveness of comprehensive psychological intervention for nonadherence to medical treatment: a case series. J Pediatr Psychol. 2013;38(6):649-663.

73. Mostert S, Sitaresmi MN, Gundy CM, Janes V, Sutaryo, Veerman AJ. Comparing childhood leukaemia treatment before and after the introduction of a parental education programme in Indonesia. Arch Dis Child. 2010;95(1):20-25.

74. Haase JE. The adolescent resilience model as a guide to interventions. J Pediatr Oncol Nurs. 2004;21(5):289-299; discussion 300-304.

75. Sansom-Daly UM, Wakefield CE, Bryant RA, et al. Online group-based cognitive-behavioural therapy for adolescents and young adults after cancer treatment: a multicenter randomised controlled trial of Recapture Life-AYA. BMC Cancer. 2012;12:339.

76. Wakefield CE, McLoone JK, Fleming CAK, et al. Adolescent cancer and health-related decision-making: An Australian multi-perspective family analysis of appointment attendance and involvement in medical and lifestyle choices. J Adolesc Young Adult Oncol. 2011;1(4):173-180.

77. Svarstad BL, Chewning BA, Sleath BL, Claesson C. The Brief Medication Questionnaire: a tool for screening patient adherence and barriers to adherence. Patient Educ Couns. 1999;37(2):113-124.
Clinical Oncology in Adolescents and Young Adults

\section{Publish your work in this journal}

Clinical Oncology in Adolescents and Young Adults is an international, peer-reviewed, open access journal publishing original research, reports, editorials, reviews and commentaries on all aspects of epidemiology, diagnosis and treatment of cancers in adolescents and young adults. The manuscript management system is completely

\section{Dovepress}

online and includes a very quick and fair peer-review system. Visit http://www.dovepress.com/testimonials.php to read real quotes from published authors. 